\title{
Asymptotic Giant Branch Evolution and the Initial-Final Mass Relation of Single CO White Dwarfs
}

\author{
Paola Marigo \\ Department of Astronomy, University of Padova, \\ Vicolo dell'Osservatorio 3, Padova, Italy \\ email: paola.marigo@unipd.it
}

\begin{abstract}
Combining recent mass determinations of Galactic CO white dwarfs and their progenitors with the latest evolutionary models for Asymptotic Giant Branch (AGB) stars, I review the initial-final mass relation (IFMR) of low- and intermediate-mass stars. In particular, I analyze the impact on the IFMR produced by a few critical processes characterizing the AGB phase, namely: the second and third dredge-up events, hot-bottom burning, and mass loss. Their dependence on metallicity and related theoretical uncertainties are briefly discussed.
\end{abstract}

Keywords. stars: AGB and post-AGB, white dwarfs, mass loss, carbon

\section{Introduction}

The initial-final mass relation (IFMR) links the mass of a star on the main sequence, $M_{\mathrm{i}}$, with the remnant mass, $M_{\mathrm{f}}$, of the white dwarf (WD) left at the end of its evolution, a fate common to low- and intermediate-mass stars with initial masses in the range from $0.9 \lesssim M_{\mathrm{i}} / M_{\odot} \lesssim 6-9$. The IFMR plays a significant role for a number of astrophysical issues. In fact, once $M_{\mathrm{i}}$ is known, the IFMR yields an estimate of the mass lost by the star over its entire evolutionary history, thus putting severe constraints to the efficiency of stellar winds during the AGB phase. The high-mass end of the IFMR provides an empirical test to the upper value for the initial mass, $M_{\mathrm{up}}$, of stars developing degenerate $\mathrm{CO}$ cores. In the wider framework of galaxy evolution, the IFMR is a key component in chemical evolution models of galaxies as it fixes the mass of the metal-enriched gas returned to the interstellar medium; it is also a critical ingredient when one aims at predicting the mass-to-light ratio of stellar populations, or their ages in combination with the observed WD luminosity functions. In the context of this conference on Type Ia supernovae, the knowledge of the IFMR is of basic importance since it determines the additional amount of material a WD in a binary system must accrete in order to reach the Chandrasekhar limit for carbon deflagration, as a function of the initial mass of the stellar progenitor.

Figure 1 displays the semi-empirical IFMR based on roughly sixty white dwarfs, mostly belonging to open clusters. The data show a clear positive correlation between the white dwarf mass and the mass of the stellar progenitor. Recently the IFMR has been extended into the low-mass range $\left(M_{\mathrm{i}}<2.5 M_{\odot}\right)$ thanks to inclusion of white dwarfs members of common proper motion pairs (CPMP; Catalán et al. 2008), or detected in old open clusters (Kalirai et al. 2008). Deriving the semi-empirical IFMR requires the intensive use of theoretical models, i.e. stellar isochrones and white dwarf cooling sequences, which unavoidably brings along all related uncertainties, e.g. assessment of cluster's age and metallicity, amount of convective overshooting, thickness of the white dwarf $\mathrm{H} / \mathrm{He}$ layers, 


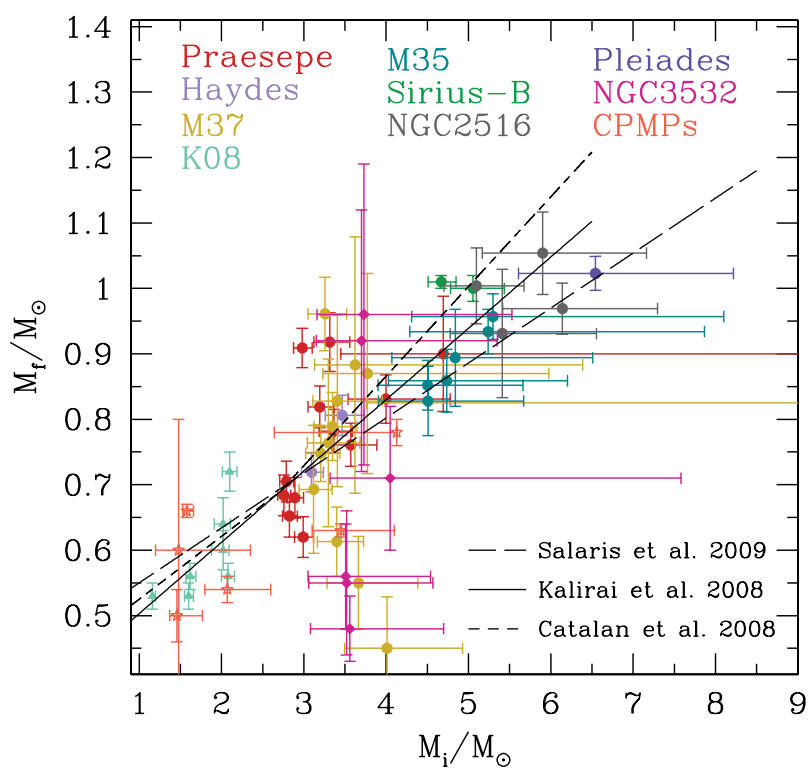

Figure 1. Semi-empirical IFMR based on the compilation by Ferrario et al. (2005), Kalirai et al. (2008), and Catalán et al. (2008). Solid lines are linear fits provided by various authors quoted in the plot.

chemical composition of the degenerate core (He, CO, ONe). A thorough analysis of all these issues can be found in Salaris et al. (2009).

Looking at Figure 1 two features are worth being noticed, namely i) a possible change of slope in the IFMR at $M_{i} \sim 3.5-4.0 M_{\odot}$ with a flattening towards larger masses, and ii) the fact the most massive white dwarfs have masses around $1.0-1.1 M_{\odot}$, quite far from the Chandrasekhar limit of $1.4 M_{\odot}$.

As far as the white dwarf mass distribution is concerned, our present knowledge relies on large samples of spectroscopically-identified objects (PG survey: Liebert et al. (2005), Sloan Digital Sky Survey: Kepler et al. (2007)). According to the SDSS data, the distribution shows the well-known prominent peak at $0.58 M_{\odot}$, a secondary peak at $0.38 M_{\odot}$, and an extended tail at larger masses, with $\sim 11 \%$ of detected white dwarfs being more massive than $0.8 M_{\odot}$. While the major peak is commonly associated to CO WDs left by AGB stars at the end their evolution, the lighter peak should be ascribed to He WDs produced by the evolution of close binary systems (Iben \& Tutukov 1993).

\section{Critical Masses}

In the framework of stellar evolution, it is convenient to group stars in different ranges of initial masses depending on their final fate (see Herwig 2005). Stars with $M_{\mathrm{i}} \leqslant M_{\text {up }}$ develop an electron-degenerate CO core after central He-burning, then experience the canonical AGB phase and eventually leave CO white dwarfs as dark remnants. Stars with $M_{\mathrm{up}} \leqslant M_{\mathrm{i}} \leqslant M_{\text {mas }}$ are able to ignite carbon in mildly-degenerate conditions, leading to the formation of O-Ne-Mg cores. They will proceed through the so-called super-AGB phase, ending as either ONe white dwarfs or electron-capture supernovae, depending on the core mass. Both critical masses $M_{\text {up }}$ and $M_{\text {mas }}$ heavily depend on uncertain aspects of stellar models; in particular present predictions locate $M_{\text {up }}$ in the range $\sim 6-8 M_{\odot}$ (Siess 2007). 


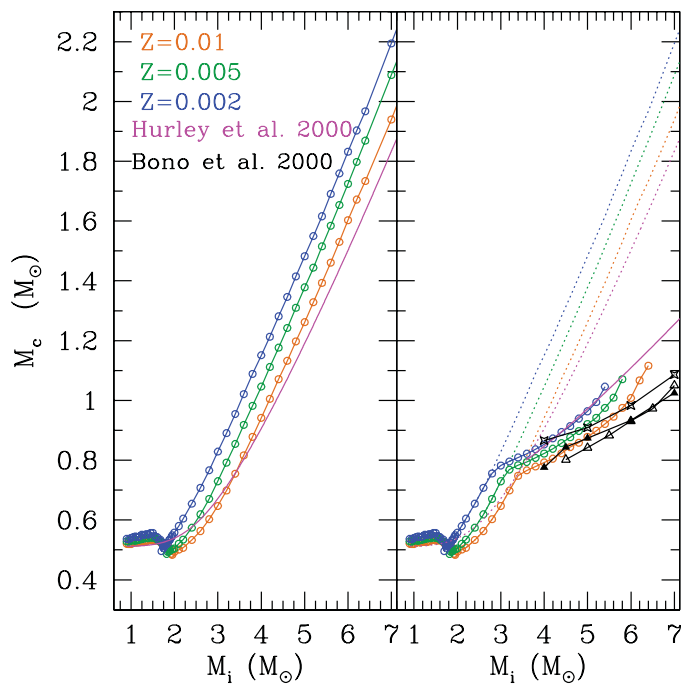

Figure 2. Core mass as a function of the initial stellar mass, at the onset of the E-AGB (left-hand side panel), and after the second dredge-up (right-hand side panel). Results from various sets of models are shown. Circles refer to computations for different metallicities, performed with the Padova stellar evolution code (Bressan et al., in prep.).

\section{Stellar Evolution of CO White Dwarf Progenitors}

The Asymptotic Giant Branch (AGB) is the last phase of the evolution of low- and intermediate mass-stars in the range $0.9 \lesssim M_{\mathrm{i}} / M_{\odot} \leqslant M_{\mathrm{up}}$. We refer to (Herwig 2005) for a comprehensive review of this phase. The internal structure of an AGB star consists of an electron-degenerate core surrounded by two nuclear burning shells, the He-burning shell and the H-burning shell, which alternate as energy sources for the star. A thin (both in mass and radius) radiative inert buffer $\dagger$ separates the nuclear region from the convective chemically-homogeneous mantle extending up to the atmosphere.

\subsection{The Early-AGB Phase and the Second Dredge-up}

Following central He-exhaustion the He-burning shell is established, and the star starts to evolve at increasing luminosity while approaching its Hayashi line for the second time during its life. During the initial stages, the so-called early-AGB (E-AGB), the H-shell is extinguished and the inner edge of the convective envelope moves inward. In stars more massive than $3.5-4.0 M_{\odot}$ the second dredge-up takes place, with notable effects both on the surface composition and internal structure of the star. The products of complete H-burning are brought up to the surface, mainly ${ }^{4} \mathrm{He}$ and ${ }^{14} \mathrm{~N}$, while ${ }^{12} \mathrm{C}$ and ${ }^{16} \mathrm{O}$ are depleted at the surface. At the same time the mass coordinate of the $\mathrm{H}$-exhausted core is shifted inward. The significant reduction of the core in massive E-AGB stars is illustrated in Figure 2, with final values not exceeding $M_{\mathrm{c}} \simeq 1.0-1.1 M_{\odot}$. It is tempting to interpret the change of slope in the IFMR, mentioned in Sect. 1 and present in the data of Figure 1, just as the signature of the second dredge-up taking place in more massive E-AGB stars.

\subsection{The TP-AGB Phase}

This phase is characterized by the quasi-periodic occurrence of thermal pulses, corresponding to thermal instabilities of the He-burning shell. The surface chemical composition may be significantly altered by mixing events taking place at thermal pulses, the third dredge-up, and, in most massive AGB stars $\left(M>3.5-4.0 M_{\odot}\right)$ by hot-bottom burning, i.e. hydrogen-burning in the deepest and hottest layers of the convective envelope

$\dagger$ The radiative buffer disappears in more massive AGB stars, with $M>4_{\odot}$, experiencing hot-bottom burning. 


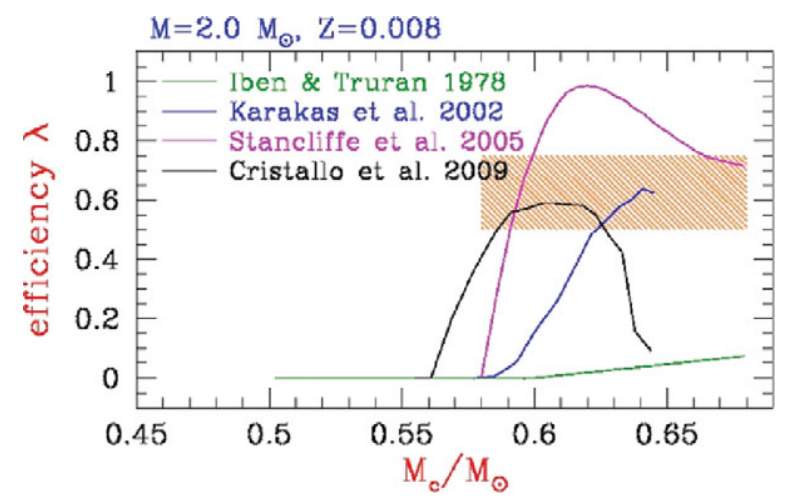

Figure 3. Efficiency of the third dredge-up, as a function of the core mass, predicted for a $2.0 M_{\odot}, Z=0.008$ model according to different authors. The dashed rectangular area brackets the typical values for $\lambda$ required to reproduce the observed carbon-star luminosity functions in the Magellanic Clouds, following the results of Groenewegen \& de Jong (1993); Stancliffe et al. (2005); Marigo \& Girardi (2007).

during quiescent stages. In the light of the latest results of AGB modeling, in the next sections I will analyze the influence of the two processes in shaping the IFMR.

The third dredge-up. During the third dredge-up the base of the convective envelope reaches across the extinct $\mathrm{H}$-shell and penetrates into the inter-shell region where the He-flash nucleosynthesis has taken place. The net consequence is the enrichment in the surface abundances of ${ }^{4} \mathrm{He}$, primary ${ }^{12} \mathrm{C}$ and ${ }^{16} \mathrm{O},{ }^{19} \mathrm{~F},{ }^{22} \mathrm{Ne},{ }^{25} \mathrm{Mg}$, and s-process elements (Iben \& Truran 1978; Cristallo et al. 2009). At the same time, the third dredge-up causes a reduction of the core mass, the entity of which is traditionally quantified via the efficiency parameter, $\lambda=\Delta M_{\mathrm{dred}} / \Delta M_{\mathrm{c}}$, given by the ratio between the dredged-up mass and the core mass increment over the preceding quiescent inter-pulse period. Despite being a key quantity, the efficiency $\lambda$ and its dependence on stellar mass and metallicity are presently affected by large uncertainties, due to our still poor physical description of convection and mixing.

Figure 3 displays how predictions for $\lambda$ have changed over the years: while in the past very weak dredge-up characterizes low-mass AGB models, a fact designated by Iben (1981) as the "carbon star mystery" (see also Iben \& Truran 1978), present models predict larger $\lambda$, as required to reproduce observations of $\mathrm{C}$ stars in the Magellanic Clouds (Marigo et al. 1999; Marigo \& Girardi 2007). We also note that results may differ considerably from author to author, which reflects the critical dependence of the third dredge-up on technical and numerical details (see Frost \& Lattanzio (1996), Mowlavi (1999) for thorough analyses).

While $\lambda$ values for low-mass stars are still quite heterogeneous, there is a general agreement among authors in predicting $\lambda \approx 1$ for more massive AGB stars, with $M_{\mathrm{i}}>$ 3-4M $\odot$ (Karakas et al. 2002; Herwig 2004; Ventura \& D'Antona 2005; Weiss \& Ferguson 2009). A typical example is illustrated in Figure 4. This implies a small increase, or even the nearly invariance of the core mass during the TP-AGB, so that the remnant mass would practically coincide with the core mass after the $2^{\text {nd }}$ dredge-up.

Hot-bottom burning. This process is expected to affect crucially the evolution of all AGB stars with large core masses, $M_{\mathrm{c}}>0.8 M_{\odot}$, and sufficiently massive envelopes, $M_{\mathrm{env}}=M-M_{\mathrm{c}}>2 M_{\odot}$. The minimum mass for the occurrence of HBB is a function of metallicity $Z$, so that lower $Z$ favors the onset of HBB at lower stellar masses (Ventura \& D'Antona 2005). HBB produces two main evolutionary effects, namely i) it makes the stars brighter than expected by the classical $M_{\mathrm{c}}-L$ relation Böcker \& Scönberner 1991), and ii) it enriches the surface chemical composition with nuclei synthesized by the C-N-O, Ne-Na, and Mg-Al cycles (Forestini \& Charbonnel 1997).

Figure 5 shows the pre-flash quiescent luminosity as a function of the core mass for a few full stellar TP-AGB models of different mass and metallicity Karakas et al. (2002). The 

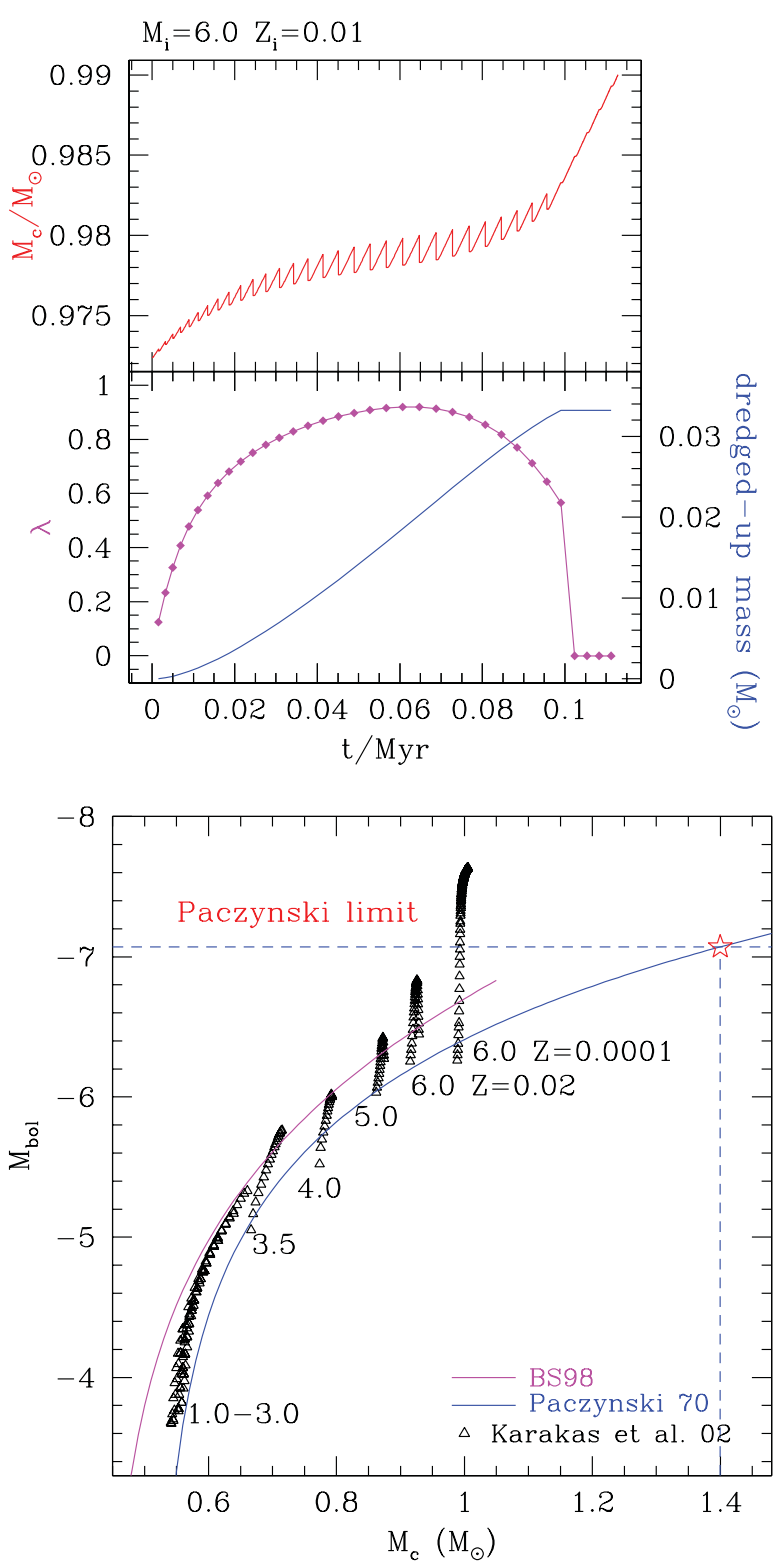

Figure 4. Top panel: Typical evolution of the core mass as a function of time during the whole TP-AGB evolution of an intermediate-mass experiencing very deep third dredge-up and HBB as well. Note the saw-tooth trend due to the periodic occurrence of the dredge-up events. Bottom panel: Evolution of the efficiency of the $3^{\text {rd }}$ dredge-up (from Karakas et al. 2002) and cumulative dredged-up mass.
Figure 5. Luminosity as a function of the core mass along the TP-AGB. Sequences marked by triangles refer to the pre-flash luminosity maximum of TP-AGB stars with various initial masses, according to Karakas et al. (2002) full evolutionary calculations. Solid lines are fit $M_{\mathrm{c}}-L$ relations from Paczyński (1970) and Boothroyd \& Sackmann (1988).

most massive stars are characterized by the HBB over-luminosity, i.e. a steep luminosity increase above the $M_{\mathrm{c}}-L$ relation, which is eventually recovered as soon as the envelope mass is drastically reduced by stellar winds. Note that the $M_{\mathrm{i}}=6 Z=0.0001$ model is expected to overcome the classical Paczynski limit $\dagger$, which in fact cannot be considered a physical limit anymore.

HBB and third dredge-up interrelate with each other in a complex fashion: on one side $\mathrm{HBB}$ delays or even prevents the formation of massive $\mathrm{C}$ stars by converting the dredgedup carbon into nitrogen, on the other side the structural cooling caused by the enhanced

$\dagger$ Traditionally the Paczynski limit corresponds to the maximum luminosity that an AGB star complying with the $M_{\mathrm{c}}-L$ relation may reach when its core mass has grown up to the Chandrasekhar limit, $M_{\mathrm{c}} \simeq 1.4 M_{\odot}$ 

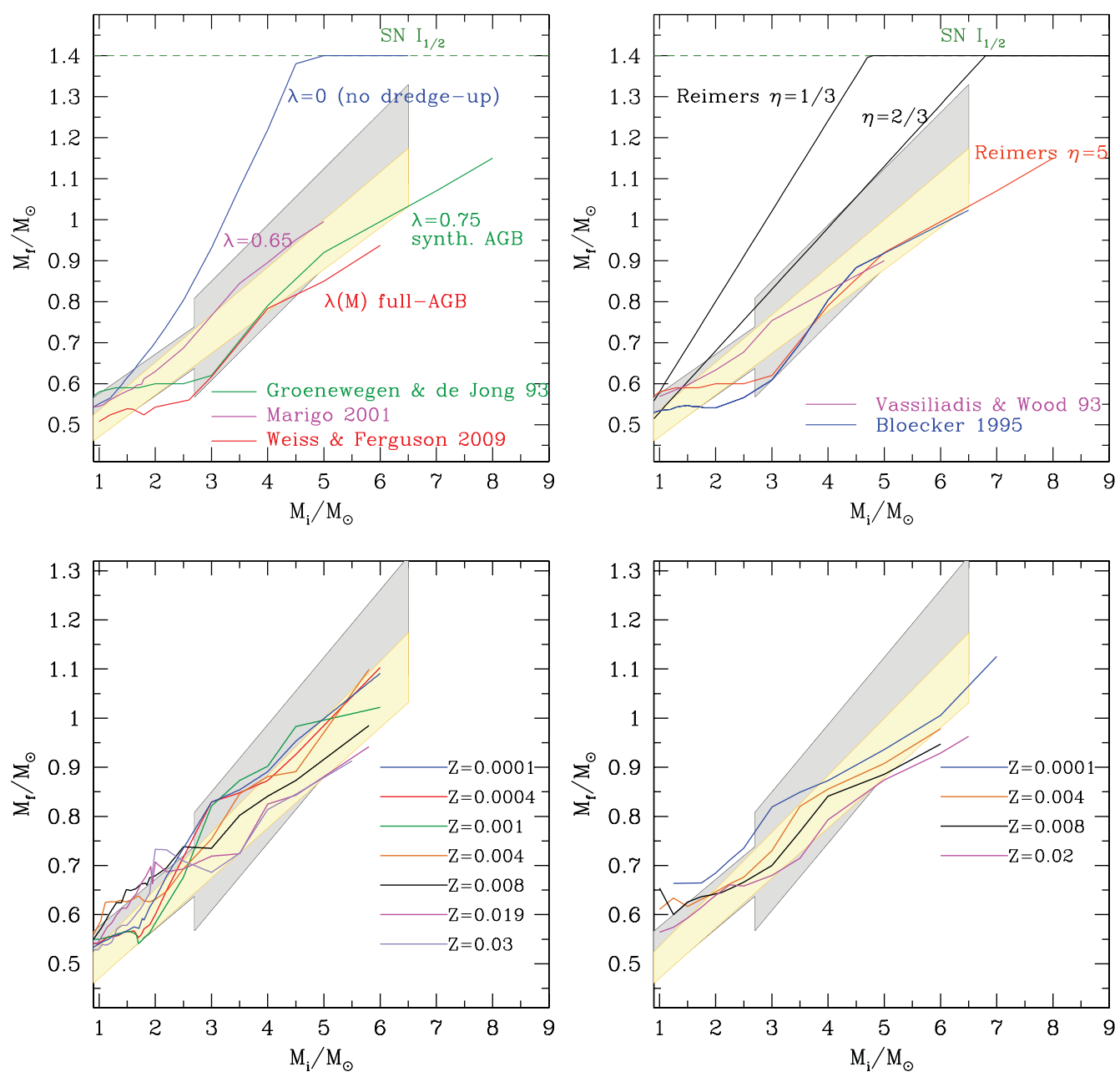

Figure 6. Dependence of the predicted IFMR on basic parameters, namely: efficiency of the third dredge-up (top left-hand side panel), the assumed mass-loss rates (top right-hand side panel), and metallicity (left bottom panel: Marigo \& Girardi (2007); right bottom panel: Karakas et al. (2002)).

C-rich opacity may weaken or even extinguish HBB (Marigo 2007; Ventura \& Marigo 2009, 2010).

\subsection{Predicted IFMR: Basic Dependencies}

It is clear that the IFMR is the result of the complex interplay among different processes, such as dredge-up events and mass loss, and their dependence on stellar mass and metallicity. Even if it is impossible to disentangle the individual role of each factor involved, Figure 6 attempts to summarize a few general trends that can be extract from AGB evolutionary models.

Dependence on the $3^{\text {rd }}$ dredge-up. The more efficient the $3^{\text {rd }}$ dredge-up, the lower is the effective increase of the core mass during the TP-AGB phase. In the extreme case $\lambda \simeq 1$, the final mass would correspond the core mass at the onset of the TP-AGB phase. The semi-empirical IFMR seems to support a modest increase of the core mass during the TP-AGB. In fact, current AGB models predict values of the core mass at the first thermal pulse which are already inside the observed width $\Delta M_{\mathrm{f}}$ of the semi-empirical 
IFMR (Karakas et al. 2002; Marigo \& Girardi 2007). Moreover, by assuming no dredgeup, we expect that intermediate mass stars would be able to reach the Chandrasekhar limit and thus explode as $\mathrm{SN} \mathrm{I}_{1 / 2}$. However, the semi-empirical IFMR indicates that at $M_{i} \simeq 5-7 M_{\odot}$ the white dwarf masses are around $1 M_{\odot}$, in any case not close to the limiting mass for $\mathrm{C}$ deflagration.

Dependence on mass loss. The larger the mass-loss rates, the shorter is the duration of the TP-AGB phase, hence the lower is the mass of the remnant. First of all, we note that barely extending to the AGB phase the classical Reimers law, commonly used (with a parameter $\eta \simeq 0.3-0.4$ ) for stars on the RGB, would again make intermediatemass stars proceed through the $\mathrm{SN} \mathrm{I}_{1 / 2}$ channel (provided that the third dredge-up $\lambda<1$ ). Synthetic AGB models (Groenewegen \& de Jong 1993) have proved that $\eta \simeq 5$ would be required on the AGB in order to match both the observed IFMR and carbon star luminosity functions on the Magellanic Clouds. However, over the years, it has become clear that the Reimers law is not suitable to describe the efficiency of mass loss on the AGB, as this formula does not account for the observed steep increase of the mass-loss rate at increasing luminosity, as shown by studies of mass-losing pulsating AGB stars Vassiliadis \& Wood (1993). Many other formalisms/recipes for AGB mass loss have been proposed, either semi-empirical (van Loon et al. 2005; Groenewegen et al. 1998; Vassiliadis \& Wood 1993), or derived from hydrodynamic dust-driven pulsationassisted wind models (Mattsson et al. 2010; Wachter et al. 2008, 2002; Arndt et al. 1997; Bloecker 1995; Bowen \& Willson 1991; Bedijn 1988).

Dependence on metallicity. A quite firm result of stellar evolution is that stars enter the TP-AGB phase with core masses that are larger at decreasing metallicity $Z$, as a result of the previous central He-burning phase. How the subsequent TP-AGB evolution shapes the IFMR as a function of $Z$ is the result of the overall dependence on metallicity of all processes involved (e.g. mass loss efficiency, depth of $3^{\text {rd }}$ dredge-up, HBB overluminosity), which is still not robustly assessed. As an example we show in Figure 6 two sets of predicted IFMRs for several metallicities, taken from the calculations by Marigo \& Girardi (2007) (left panel), and Karakas et al. (2002) (right panel). We see that while in the former set the dependence on $Z$ is not monotonic over the whole range of stellar masses, in the latter more massive white dwarfs are always expected at decreasing $Z$. This difference is likely due to differences in the description of mass loss and of molecular opacities $\dagger$ of C stars, coupled to the current surface C/O in Marigo \& Girardi (2007), frozen to a scaled-solar mixture in Karakas et al. (2002).

\section{Acknowledgements}

P.M. acknowledges financial support from contract ASI-INAF n. I/009/10/0.

\section{References}

Arndt, T. U., Fleischer, A. J., \& Sedlmayr, E. 1997, A\&A, 327, 614

Bedijn, P. J. 1988, A\&\&A, 205, 105

Blöcker, T. \& Schönberner, D. 1991, A\&A A, 244, L43

Bloecker, T. 1995, A\& A, 297, 727

Boothroyd, A. I. \& Sackmann, I.-J. 1988, ApJ, 328, 641

$\dagger$ A useful web-interface for computing accurate Rosseland mean opacities for any chemical mixture is available at http://stev.oapd.inaf.it/aesopus (Marigo \& Aringer 2009). 
Bowen, G. H. \& Willson, L. A. 1991, ApJL, 375, L53

Catalán, S., Isern, J., García-Berro, E., Ribas, I., Allende Prieto, C., \& Bonanos, A. Z. 2008, $A \mathscr{G} A, 477,213$

Cristallo, S., Straniero, O., Gallino, R., Piersanti, L., Domínguez, I., \& Lederer, M. T. 2009, ApJ, 696, 797

Ferrario, L., Wickramasinghe, D., Liebert, J., \& Williams, K. A. 2005, MNRAS, 361, 1131

Forestini, M. \& Charbonnel, C. 1997, A\& $A S, 123,241$

Frost, C. A. \& Lattanzio, J. C. 1996, ApJ, 473, 383

Groenewegen, M. A. T. \& de Jong, T. 1993, A\& A, 267, 410

Groenewegen, M. A. T., Whitelock, P. A., Smith, C. H., \& Kerschbaum, F. 1998, MNRAS, 293, 18

Herwig, F. 2004, ApJ, 605, 425

- 2005, ARAA, 43, 435

Iben, I., Jr. 1981, $A p J, 246,278$

Iben, I., Jr. \& Truran, J. W. 1978, ApJ, 220, 980

Iben, I., Jr. \& Tutukov, A. V. 1993, ApJ, 418, 343

Kalirai, J. S., Hansen, B. M. S., Kelson, D. D., Reitzel, D. B., Rich, R. M., \& Richer, H. B. 2008, ApJ, 676, 594

Karakas, A. I., Lattanzio, J. C., \& Pols, O. R. 2002, PASA, 19, 515

Kepler, S. O., Kleinman, S. J., Nitta, A., Koester, D., Castanheira, B. G., Giovannini, O., Costa, A. F. M., \& Althaus, L. 2007, MNRAS, 375, 1315

Liebert, J., Bergeron, P., \& Holberg, J. B. 2005, ApJS, 156, 47

Marigo, P. 2007, A\&SA, 467, 1139

Marigo, P. \& Aringer, B. 2009, $A \mathscr{E} A, 508,1539$

Marigo, P. \& Girardi, L. 2007, $A \& A, 469,239$

Marigo, P., Girardi, L., \& Bressan, A. 1999, A\& A, 344, 123

Mattsson, L., Wahlin, R., \& Höfner, S. 2010, A\&A, 509, A14+

Mowlavi, N. 1999, A\& $A, 344,617$

Paczyński, B. 1970, Acta Astronomica, 20, 287

Salaris, M., Serenelli, A., Weiss, A., \& Miller Bertolami, M. 2009, ApJ, 692, 1013

Siess, L. 2007, $A \& A, 476,893$

Stancliffe, R. J., Izzard, R. G., \& Tout, C. A. 2005, MNRAS, 356, L1

van Loon, J. T., Cioni, M.-R. L., Zijlstra, A. A., \& Loup, C. 2005, A\&A, 438, 273

Vassiliadis, E. \& Wood, P. R. 1993, ApJ, 413, 641

Ventura, P. \& D'Antona, F. 2005, A\& $A, 431,279$

Ventura, P. \& Marigo, P. 2009, MNRAS, 399, L54

- 2010, MNRAS, 408, 2476

Wachter, A., Schröder, K.-P., Winters, J. M., Arndt, T. U., \& Sedlmayr, E. 2002, A\&SA, 384, 452

Wachter, A., Winters, J. M., Schröder, K.-P., \& Sedlmayr, E. 2008, AE\&A, 486, 497

Weiss, A. \& Ferguson, J. W. 2009, A\& A, 508, 1343 